\title{
Conversion of waste cooking oil by rhodococcal lipase immobilized in gellan gum
}

\author{
Maegala Nallapan Maniyam ${ }^{1,2 *}$, Hazeeq Hazwan Azman ${ }^{1,2}$, Hasdianty Abdullah ${ }^{1,3}$, and Nor \\ Suhaila Yaacob ${ }^{1,2}$ \\ ${ }^{1}$ Institute of Bio-IT Selangor, Universiti Selangor, Jalan Zirkon A7/A, Seksyen 7, 40000 Shah Alam, \\ Selangor Darul Ehsan, Malaysia. \\ ${ }^{2}$ Centre for Foundation and General Studies, Universiti Selangor, Jalan Timur Tambahan, 45600 \\ Bestari Jaya, Selangor Darul Ehsan, Malaysia. \\ ${ }^{3}$ Faculty of Engineering and Life Sciences, Universiti Selangor, Jalan Timur Tambahan, 45600 \\ Bestari Jaya, Selangor Darul Ehsan, Malaysia.
}

\begin{abstract}
Recently, the application of lipase enzyme as biocatalyst in the conversion of waste cooking oil (WCO) to free fatty acids and glycerol has been trending well. Therefore, the present study attempts to use WCO which is found in abundance in Malaysia as the substrate for halal microbial lipase conversion to glycerol which can be exploited in the food industry. The workability of free lipase for WCO conversion, however suffers severely due to potential denaturation of the enzyme and extended reaction time. Thus, this study embraced the immobilization method to encapsulate crude lipase extracted from Rhodococcus pyridinivorans strain UCC 0009 in gellan gum and calcium alginate, respectively and compared their ability for WCO conversion to free crude lipase. The gellan gum and calcium alginate-immobilized crude lipase evidently exhibited greater WCO conversion, demonstrating 2.18-fold and 1.61-fold enhanced lipase activity, respectively in comparison to free crude lipase. The repeated reuse of the gellan gum-immobilized crude lipase maintained reasonable lipase activity for 9 cycles, retaining an average $85 \%$ WCO conversion for the first seven cycles and $67 \%$ conversion in the subsequent batches. Thus, the immobilized halal lipase can be foreseen as a green substitute to chemical catalyst for WCO conversion which meets the worldwide demand for clean technologies.
\end{abstract}

\section{Introduction}

Many industries namely textiles, medicines, leather, food and detergents have extensively accommodated the use of enzymes in their practices since the emergence of enzymology [1]. It is estimated that by 2023 , the global industrial enzyme market will escalate rapidly by $4.9 \%$ to reach US\$7 billion [1]. With the transformation of the chemical processes to enzymatic processes for goods and commodities assembly, the gradual adaptation of industrial-scale production processes over the years may contribute largely to this growth

\footnotetext{
*Corresponding author: maegala@unisel.edu.my; maegala_81@yahoo.com
} 
[2]. The broad biochemical diversity of microorganisms and the achievability of large-scale production are the main factors that turn microorganisms into the most commonly used source for the production of industrial enzymes [1]. In addition, enzymes have been increasingly used in several industries and therefore, it is a pressing task to find new microbial isolates that can produce functional enzymes to meet this growing demand [3].

Lipase is a multifunctional, industrially important enzyme that can be produced economically from microorganisms and used as a biocatalyst in a variety of industrial applications, including food processing technology, oil processing, medicine and biodegradation [4]. Microbial lipase is also important because it is easy to ferment and purify and thus presents itself as one of the most common sources for biodiesel production through transesterification [4]. However, the utilization of free lipase may carry some disadvantages such as high cost, low stability and long reaction time [5]. Therefore, lipase immobilisation on suitable support is the key parameter to achieve enhanced stability, reusability, easy product separation and recovery, reduced cost for lipase production and improved control of reactions [6].

Among the four immobilization techniques namely entrapment, physical adsorption, covalent binding and cross-linking, the entrapment technique emerged as the most beneficial approach since this technique is straightforward, requires low cost of operation, can be performed under mild reaction conditions, high recovery of activity and allows effortless diffusion of substrates through the immobilization matrix pore [5]. In addition, the entrapment technique does not use physical force and chemical reaction and by this means supports stable enzyme conformation for a longer period of time [5]. The utilization of hydrogels in particular to immobilize the enzyme offers many benefits such as the ability to absorb water and swell readily without dissolving and sustaining the three-dimensional structure of enzyme [5].

Sphingomonas elodea secretes an anionic heteropolysaccharide known as gellan gum which was discovered way back in 1980 and has ever since been the subject of interest in multiple research areas [7]. In most cases, gellan gum has been extensively employed as biological material and as an emulsifier, thickening and suspending medium in the food and cosmetic industries [7]. Gellan gum appears to be a finest biological polymer for entrapment of active enzymes. This immobilization matrix has better rheological characteristics in comparison to that of agar and carrageenan gels when assessed at similar concentrations [8]. Besides, the hydrogels are able to sustain stability over a broad $\mathrm{pH}$ range which is from 2 to 10 [9]. Gellan gum can be used as a simple matrix for enzyme immobilization due to its polyelectrolyte property, hydrophilicity, emulsification attributes, its non-hazardous features, biocompatibility and biodegradability [7].

Malaysia is one of the largest waste cooking oil (WCO) producing countries contributing approximately 1 million tonnes per year [10]. Therefore, it is only appropriate to exploit WCO for conversion to beneficial products due to its plentiful presence in Malaysia [10]. Lipase catalyses the conversion of WCO to biodiesel and glycerol. Since the use of biodiesel is still quite limited in Malaysia, the focus to produce glycerol which has many applications particularly in the food industry is highly desired. Porcine pancreatic lipases are commonly used in the food industry due to numerous advantages such as costeffective, readily available and could withstand high temperature among others [10]. However, this becomes a grave issue to the Muslim majority population in Malaysia since commercial lipases are usually obtained from porcine which is deemed non-halal. Therefore, the present study investigated the viability of using different entrapment matrices for the immobilization of crude lipase obtained from Rhodococcus pyridinivorans strain UCC 0009 for successful conversion of WCO. In addition, the crude lipase immobilized in gellan gum was tested for repeated use. This is the first study which reports the utilization of immobilized crude lipase obtained from tropical rhodococci for WCO 
conversion to the best knowledge of the authors. The authors are very encouraged that the findings from the present study will provide the important information for potential largescale application of the immobilization method for WCO conversion to glycerol using halal microbial lipase.

\section{Method}

\subsection{Chemicals}

Gellan gum and sodium alginate were supplied by Sigma (USA). All other chemicals and reagents were of analytical grade and purchased from either Systerm (Malaysia), Fisher Scientific (Singapore) or Merck (Germany) and were subsequently used without further purification.

\subsection{Preparation of seed culture}

Rhodococcus pyridinivorans strain UCC 0009 with a gene accession number of MN56068 [11] was obtained from the culture collection unit, Universiti Selangor. The strain was streaked on nutrient agar plates $(20 \mathrm{~g} / \mathrm{L})$ and the plates were kept in an incubator (MEMMERT 108L Incubator INB500, Germany) at $30{ }^{\circ} \mathrm{C}$ for 36 hours. An amount of 100 $\mathrm{mL} 8 \mathrm{~g} / \mathrm{L}$ nutrient broth was then inoculated with a loopful of Rhodococcus pyridinivorans strain UCC 0009 and left to agitate at $30^{\circ} \mathrm{C}$ and $160 \mathrm{rpm}$ for 24 hours (Jeio Tech SI-600R, Korea). This culture served as the inoculum (seed culture) with an optical density between 0.6 to 0.8 (the active exponential phase of growth curve).

\subsection{Production of lipase from Rhodococcus pyridinivorans strain UCC 0009}

Minimal salt medium (MSM) following the formulation described by Nallapan Maniyam et al. [12] was established in the present study. An inoculum concentration of $8 \%(\mathrm{v} / \mathrm{v})$ was redistributed into $250 \mathrm{~mL}$ conical flask containing $50 \mathrm{~mL}$ MSM medium and the flask was placed in an incubator shaker for 24 hours at $30^{\circ} \mathrm{C}$ and $160 \mathrm{pm}$.

\subsection{Protein extraction, quantification and determination of crude lipase activity}

The whole cells of Rhodococcus pyridinivorans strain UCC 0009 was pulled together after 24 hours of incubation period through centrifugation at $4400 \mathrm{rpm}$ for 30 minutes (Eppendorf 5702R, South Asia). Potassium phosphate buffer (0.05 M, pH 7) was used to wash the remaining cell pellet after centrifugation and meticulous elimination of the supernatant. The washed cells were then resuspended in $2 \mathrm{~mL}$ of fresh potassium phosphate buffer and placed in a pre-chilled mortar for cryogenic grinding adhering to the protocols established by Nallapan Maniyam et al. [12]. The protein content of the extracted lipase and its activity were determined accordingly following previously published methods [10].

\subsection{Immobilization of crude lipase}

Crude lipase (5 mg) was immobilized in calcium alginate [12] and gellan gum [9] resulting in the formation of 50 beads, respectively. The control beads were prepared in the same manner, however without the inclusion of crude lipase. Bradford's method was employed 
to quantify the unbound protein gathered in the supernatant and the washing solution which was addressed as cell leakage. Equation 1 elucidates the formula to determine immobilization yield.

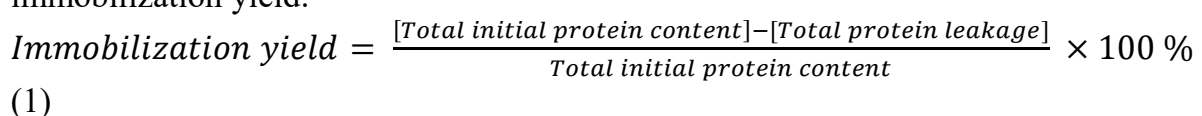

\subsection{Conversion of WCO and repeated use of immobilized crude lipase}

A restaurant located nearby the university supplied the WCO which was sifted to separate the solid food before use in the experimental protocols. Fifty beads of immobilized lipase (5 $\mathrm{mg}$ ) in calcium alginate and gellan gum, respectively were added to $5 \mathrm{~mL} \mathrm{WCO}$ and $20 \mathrm{~mL}$ $0.05 \mathrm{M}$ phosphate buffer. The reaction mixture in triplicate was incubated at $160 \mathrm{rpm}$ and $30{ }^{\circ} \mathrm{C}$ for 24 hours. After the reaction was concluded, the oil face containing free fatty acid was parted through centrifugation at $4400 \mathrm{rpm}$ for 30 minutes. The quantification of free fatty acid was then carried out using the oil phase based on the standard titration method [13] for acid value determination. This procedure was repeated with $5 \mathrm{mg}$ free crude lipase in triplicate and the control system in the absence of crude lipase and control beads, respectively.

A colander was used to filter the immobilized beads in gellan gum from the medium containing WCO and phosphate buffer after 24 hours of incubation period. The recovered beads were subjected to rinsing using $0.9 \%(\mathrm{w} / \mathrm{v})$ sodium chloride and immersed in $0.3 \mathrm{M}$ calcium chloride solution for 2 hours. After 2 hours, the beads were taken from the calcium chloride soliton and repeatedly washed with sodium chloride. The washed beads were then supplemented in fresh medium containing $5 \mathrm{~mL} \mathrm{WCO}$ and $20 \mathrm{~mL} 0.05 \mathrm{M}$ phosphate buffer and the protocols were duplicated to determine the acid value for the conversion of WCO. This process was repeated for several cycles until the WCO conversion declined considerably.

\subsection{Statistical analysis}

The determination of error bars which was obtained from the Excel application were carried out using triplicate data for each experimental method. A one-way ANOVA test with $95 \%$ confidence interval was established to examine the difference between group aided by the SPSS software version 20.0 ( $\mathrm{p}<0.05$ is regarded as statistically significant). The most optimum value for each experimented factor were verified using the Duncan test via the post-hoc analysis (denoted by different letters).

\section{Results and discussion}

The field of green chemistry favours the use of enzymatic bioconversion specifically the crude enzyme due to its attractive attributes namely high activity, high specificity and formation of less toxic metabolites which prompted the present study. The crude lipase was extracted from Rhodococcus pyridinivorans strain UCC 0009 via the cryogenic grinding in liquid nitrogen in view of the fact that the technique is facile, rapid and low-cost [14]. Negligible conversion of WCO in the control system (with control beads and without the addition of crude lipase, respectively) was observed in all experiments carried out in the present study.

\subsection{Conversion of WCO by crude lipase and immobilized crude lipase}


Table 1 shows the comparative data on WCO conversion by free crude lipase and different carrier matrices of immobilized crude lipase extracted from Rhodococcus pyridinivorans strain UCC 0009.

Table 1. Conversion of WCO by free and immobilized rhodococcal crude lipase.

\begin{tabular}{|c|c|c|c|}
\hline Type of carriers & WCO Conversion (\%) & Immobilization Yield (\%) & Protein Leakage (mg) \\
\hline Free cell & $38^{\mathrm{c}} \pm 1 \%$ & Not Applicable & Not Applicable \\
\hline Calcium Alginate & $61^{\mathrm{b}} \pm 2 \%$ & $77^{\mathrm{b}} \pm 1 \%$ & $1.15 \pm 0.98$ \\
\hline Gellan Gum & $83^{\mathrm{a}} \pm 1 \%$ & $88^{\mathrm{a}} \pm 2 \%$ & $0.60 \pm 0.14$ \\
\hline
\end{tabular}

The control system was established without the presence of crude lipase and immobilized crude lipase, respectively. Equal amounts of free and immobilized crude lipase were added to a solution containing $5 \mathrm{~mL}$ WCO and $20 \mathrm{~mL}$ of $0.05 \mathrm{M}$ phosphate buffer to initiate the conversion. The incubation was at $30{ }^{\circ} \mathrm{C}$ for 24 hours. WCO conversion, immobilization yield and protein leakage were means of triplicate samples \pm standard errors. Statistically significant differences $(p<.05)$ were observed among the tested carriers (different letters with $\mathrm{a}=$ most optical and $\mathrm{c}=$ least optimal).

The lowest conversion of WCO was evidently observed with free crude lipase extracted from Rhodococcus pyridinivorans strain UCC 0009, yielding $38^{c} \pm 1 \%$ conversion (different letters indicate significant differences in the tested factors statistically). WCO can be presented as toxic since the properties of healthy vegetable oils at room temperature can change substantially upon repeated heating [15]. Therefore, this condition eventually made the crude lipase to be susceptible to the hazardous WCO.

The ability of crude lipase extracted from the tropical rhodococci for WCO conversion can be improved with the immobilization technique. The immobilized crude lipase provides higher catalytic activity and stability, alleviates separation challenges, facilitates scale-up and promotes green approach through recycling of the beads [16]. In the present study, the most generic immobilization method namely the gel entrapment using gellan gum and calcium alginate was developed, respectively. These matrices were nonhazardous, cheap, readily available, biocompatible and flexible to modify which provided stability and protection to the crude extract (crude lipase in this case) and thus, led to their selection as the immobilization support matrix [17]. Fascinatingly, substantially greater WCO conversion $(p<0.05)$ was observed with immobilized crude lipase in both matrices compared to that of the non-immobilized counterpart. The improved WCO conversion may be attributed to enhanced rigidity of the enzyme which shielded it from unfolding and avoided the conformational transition of the enzyme [18].

When the crude lipase immobilized in calcium alginate was allowed to come in contact with $5 \mathrm{~mL}$ WCO in $20 \mathrm{~mL}$ phosphate buffer, a $61^{\mathrm{b}} \pm 2 \%$ conversion of WCO was recorded, documenting a $27 \%$ lower WCO conversion in comparison to crude lipase immobilized in gellan gum. Calcium alginate matrix has been used extensively to immobilize whole cells of microorganisms or their enzymes for bioremediation purposes due to their many attractive qualities. Nonetheless, in the present study, a moderate amount of cell leakage amounting to $1.15 \pm 0.98 \mathrm{mg}$ was observed which indicated that the calcium alginate matrix was quite vulnerable to disintegration which may be due to insufficient cross-linking ion. Further study focusing on the optimization of calcium alginate bead formulation such as allowing the beads to cure longer in calcium chloride solution will be carried out to rectify this challenge.

The employment of the crude lipase extracted from Rhodococcus pyridinivorans strain UCC 0009 and immobilized in gellan gum surfaced as the most proficient method in the 
conversion of WCO yielding $83^{\mathrm{a}} \pm 1 \%$ conversion, a considerable increase in the extent of WCO conversion by $36 \%$ and $118 \%$ compared to the use of calcium alginate-immobilized crude lipase and free crude lipase in WCO conversion, respectively. Joshi et al. [19] reported that WCO conversion rate by immobilized lipase was higher than free lipase which was similar to the findings observed in the present study. Joshi et al. [19] reported that WCO conversion rate by immobilized lipase was higher than free lipase which was similar to the findings observed in the present study. In addition, a study reported that the immobilization approach increased the conversion rate of vegetable oil by promoting the stability of lipase at higher temperature and in the presence of tert-butanol compared to the free enzyme through the prevention of premature deactivation of lipase [20]. The employment of gellan gum beads for effective WCO conversion was found to be favourable since the method provides chemical, mechanical and thermal stability. Moreover, the utilization of gellan gum as the immobilization matrix provided an extra edge in comparison to calcium alginate. The interaction between gellan gum and ions is nonspecific which allows the gel to interact with multiple cations which is not achievable with other ion -sensitive gelling polysaccharides such calcium alginate [8].

\subsection{Repeated use of immobilized crude lipase for WCO conversion}

An experimental protocol was carried out in order to evaluate whether the rhodococcal crude lipase fixed in gellan gum can be reused for the conversion of WCO. It is important to invest in a research study that supports sustainability with minimum cost which can be achieved not only by optimum use of chemical reagents and reduced reaction period but also through reusability which increases the potential industrial application of the immobilized crude lipase in terms of economic aspects [19]. This is because compared with free crude lipase, immobilized crude lipase can be easily separated from the reaction solution and reused.

The reusability performance of the crude lipase extracted from Rhodococcus pyridinivorans strain UCC 0009 and immobilized in gellan gum which was assessed for nine consecutive cycles of WCO conversion is as shown in Figure 1. The gellan gumimmobilized crude lipase maintained its activity for seven successive cycles resulting in an average of $85^{\mathrm{a}} \pm 1 \% \mathrm{WCO}$ conversion. It was worth noting that after seven consecutive use of gellan gum-immobilized lipase, the rate of WCO conversion was only reduced by approximately $20 \%$. The immobilized crude lipase exhibited $80 \%$ conversion efficiency of its initial activity after seven batches which further reduced to a lower yield of $78 \%$ at the end of the ninth batch.

Enzyme deactivation may have largely contributed to the gradual decrease in the WCO conversion efficiency after seven succeeding cycles [21]. In addition, certain pore of the immobilized beads may be congested with the substrates or the resulting products from the conversion of WCO which may have hampered the activity of rhodococcal crude lipase immobilized in gellan gum after successive conversion cycles.

It was interesting to observe that very minimal amount of cell leakage occurred at cycles eight and nine which may be due to the washing process at the end of each batch. Reusability of immobilized crude lipase over seven cycles with relatively high WCO conversion in nine cycles using gellan gum-immobilized crude lipase is in agreement with previous report of using iron nano particles as lipase immobilization matrix [19]. Interestingly, no WCO conversion was observed in the control system confirming that the WCO conversion was indeed due to the lipase activity. The findings from the present study indicated that the crude lipase extracted from Rhodococcus pyridinivorans strain UCC 0009 and immobilized in gellan gum exhibited good reusability as biocatalysts. Therefore, these 
biocatalysts have a huge potential to be utilized in the conversion of voluminous WCO particularly in the application of continuous systems.

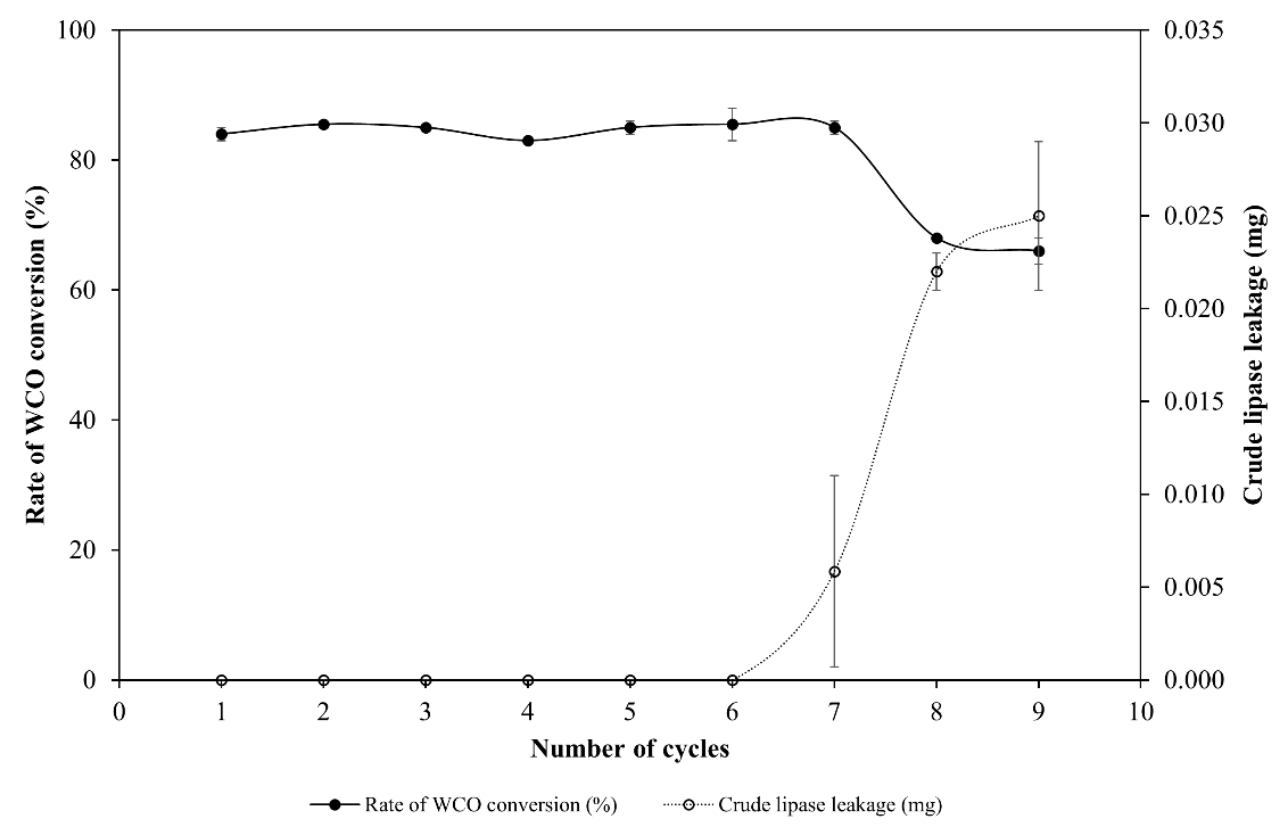

Fig. 1. The conversion of WCO by crude lipase extracted from Rhodococcus pyridinivorans strain UCC 0009 and immobilized in gellan gum for nine repeated cycles. Control was established in the absence of crude lipase in the immobilized beads. The rate of WCO conversion and crude lipase leakage represent means of triplicate samples \pm standard errors. Incubation for each cycle was at 30 ${ }^{\circ} \mathrm{C}$ and $160 \mathrm{rpm}$ for 24 hours. The $\mathrm{pH}$ of the medium was left unadjusted. Error bars represent standard error between three determinations.

\section{Conclusion}

The present study revealed that the utilization of crude lipase extracted from Rhodococcus pyridinivorans strain UCC 0009 and immobilized in gellan gum yielded in greater WCO conversion in contrast to calcium alginate-immobilized crude lipase and free crude lipase, respectively which may be possibly due to enhanced enzyme-substrate interaction. The rate of WCO conversion was higher by $36 \%$ and $118 \%$ for gellan gum-immobilized crude lipase compared to that of calcium alginate-immobilized crude lipase and free crude lipase, respectively. Moreover, rhodococcal crude lipase immobilized in gellan gum could be recycled up to nine cycles without considerable loss in the lipase activity which proved to be beneficial for up-scaling process. This study demonstrated that with further optimization using response surface methodology (RSM), the use of immobilized lipase in the conversion of WCO could improve conversion rate, reduce reaction time and lead to cost reduction in industrial practice as a result from continuous use.

\section{Acknowledgements}

This research work would not have been possible without the financial support from the Selangor State Government and Universiti Selangor (Unisel), Malaysia through the Geran Penyelidikan Negeri Selangor (GPNS-01/Unisel/18-002) research grant. 


\section{References}

1. J.M. de Oliveira, P. Fernandes, R.G. Benevides, S.A. de Assis, 3 Biotech. 10(10), 1-14 (2020)

2. P. Vashist, R. Kanchana, V.L.A Devasia, P.V. Shirodkar, U.D. Muraleedharan, Advances in Biological Science Research (Academic Press, 2019)

3. G. Banerjee, A.K. Ray, Biotechnol. Genet. Eng. Rev. 33(2), 119-143 (2017)

4. S.O. Kareem, E.I. Falokun, S.A. Balogun, O.A. Akinloye, S.O. Omeike, Beni-Suef Univ. J. Basic Appl. Sci. 9(1), 1-8 (2020)

5. P. Muanruksa, P. Dujjanutat P. Kaewkannetra, Catalysts. 10(8), 834 (2020)

6. S.W. Hassan, H.H. Abd El Latif, S.M. Ali, Front. Microbiol. 9, 2377 (2018)

7. N. Khan, Q. Husain, N. Qayyum, Int. J. Biol. Macromol. 165, 2000-2009 (2020)

8. F.N.A. Muliadi, M.I.E. Halmi, S.B.A. Wahid, S.S. Abd Gani, K. Mahmud, M.Y. Abd Shukor, Sustainability. 13(1), 1-1 (2020)

9. M.N. Maniyam, A.L. Ibrahim, A.E. Cass, Environ. Technol. 40(3), 386-398 (2019)

10. N.M. Maegala, S. Anupriya, A.H. Hazwan, Y.N. Suhaila, A. Hasdianty, IOP Conference Series: Earth and Environmental Science (IOP Publishing, 2020)

11. A. Hasdianty, Y.N. Suhaila, A.H. Hazwa, M.N. Maniyam, A.M. Fadzli, A.L. Ibrahim, Biocatal. Agric. Biotechnol. 30, 101840 (2020)

12. M. Nallapan Maniyam, A. Sundarajoo, H. Hazwan Azman, N. Suhaila Yaacob, H. Abdullah, E3S Web of Conferences (EDP Sciences, 2020)

13. N. Saifuddin, A.Z. Raziah, H. Farah, E-J Chem. 6(S1), S485-S495 (2009)

14. M. Nallapan Maniyam, F. Sjahrir, A. Latif Ibrahim, A.E. Cass, J. Environ. Sci. Health A. 50(4), 357-364 (2015)

15. C.H. Okino-Delgado, D.Z.D. Prado, R. Facanali, M.M.O. Marques, A.S. Nascimento, C.J.D.C. Fernandes, L.F. Fleuri, PloS One. 12(10), e0186246 (2017)

16. K. Li, Y. Fan, Y. He, L. Zeng, X. Han, Y. Yan, Sci. Rep. 7(1), 1-17 (2017)

17. C. Peptu, A. Savin, L.I. Atanase, K. Souidi, G. Mackenzie, P. Martin, M. Popa,. Int. J. Polym. Sci. 2017, 7610420 (2017)

18. Z. Chen, L. Liu, R. Yang, RSC Adv. 7(56), 35169-35174 (2017)

19. R. Joshi, R. Sharma, A. Kuila, Bioresour. Technol. Rep. 5, 134-140 (2019)

20. M. Cea, M.E. González, M. Abarzúa, R, J. Environ. Manage. 242, 171-177 (2019)

21. M.N. Maniyam, N.S. Yaacob, H.H. Azman, N.A. Ab Ghaffar, H. Abdullah, Biocatal. Agric. Biotechnol. 16, 569-578 (2018)

22. D. Daâssi, S. Rodríguez-Couto, M. Nasri, T. Mechichi, Int. Biodeterior. Biodegrad. 90, 71-78 (2014) 